\title{
Usage of Internet in Seeking Health Information among Medical and Dental Practitioners in Hyderabad
}

Dr. E. Mamatha MDS ${ }^{*}$, Dr. Yadav Kopula², Dr. Sheetal Akula, MDS³, Dr. Goje Vidyasagar, MDS³, T. N Abhinav, MDS 3 , Dr. Shakeel Anjum, MDS4

Post Graduate, Department of Public Health Dentistry, Sri Sai College of Dental Surgery, Vikarabad, Telangana, India 2Reader, Department of Public Health Dentistry, Sri Sai College of Dental Surgery, Vikarabad, Telangana, India ${ }^{3}$ Senior Lecturer, Department of Public Health Dentistry, Sri Sai College of Dental Surgery, Vikarabad, Telangana, India ${ }^{4}$ Professor, Department of Public Health Dentistry, Sri Sai College of Dental Surgery, Vikarabad, Telangana, India

\section{*Corresponding Author}

Dr. E. Mamatha

\section{Article History}

Received: 12.07.2019

Accepted: 24.07.2019

Published: 30.07 .2019

\begin{abstract}
Introduction: The Internet has revolutionized the way information is shared and accessed. Information retrieval is easier now than ever before due to drastic increase in rise of modern search engines. One important domain in which the Internet plays an increasing role is health information access. Methodology: A cross sectional study was conducted among 200 medical and 200 dental practitioners in Hyderabad. A pretested structured 15 item questionnaire was used to collect the data. Chi square test was used to assess the difference in internet usage among medical and dental practitioners. Results: Mean age of the practitioners was 37 years and majority of them were males. $76 \%$ dental practitioners use internet for getting updated about new materials and techniques.78\% of medical practitioners used internet to get updated about the recent advances in treatment procedures. Conclusion: There is a significant difference in the usage of internet among medical and dental practitioners.
\end{abstract}

Keywords: Internet, health information, Health practitioners

\section{INTRODUCTION}

The Internet is a global network of networks that enables computers of all kinds to directly and transparently communicate throughout the world. It is described as a global network and an 'Information Super-highway' [1]. It is defined as a world-wide broadcasting capability, a mechanism for information dissemination, and a medium for collaboration and interaction between individuals and their computers without regard to geographical location, the Internet Society 2010. The internet is indeed a major technological breakthrough of our time.

Humans group together in order to interact, cooperate, exchange ideas and influence each other. Consequently, in a very basic sense, a social network is an organized set of people consisting of two elements: human beings and the connections between them. The Internet has revolutionized the way information is shared and accessed. Information retrieval is easier now than ever before. Since the rise of modern search engines, social networks, and ubiquitous access through devices such as smart phones and tablet or laptop computers, information is available at people's fingertips almost any time of the day [2]. The Internet is currently transforming many aspects of life, especially in the ways people are accessing health information [3].

One important domain in which the Internet plays an increasing role is health information access. In recent years, the use of internet by and health care professionals has grown significantly. The advantages of applying new information and communication technologies in health care have well been established. The literature has also shown that within this broad field of new developments in health care, internet hold considerable potential value, because they enable new ways of access to and sharing of information, social support, emphasize collaboration and participation and increase individuals' connectivity $[4,5]$. Currently, up to $70 \%$ of the general public seeks health care information and advice from Internet sources, and they continue to use Web-based resources to strengthen their capacity to communicate about their health needs. Recent advances on internet technologies, particularly the

Copyright @ 2019: This is an open-access article distributed under the terms of the Creative Commons Attribution license which permits unrestricted use, distribution, and reproduction in any medium for non commercial use (NonCommercial, or CC-BY-NC) provided the original author and source are credited. 
participative internet, did transform patterns of - amongst others - health-related communication, by creating new information highways for instant communication and online feedback between healthcare professionals and patients. Thus, internet a great information equalizer, is radically transforming the way people within the healthcare sector, around the world, communicate and facilitate accurate health information to reach many people, more than any other technology or process in the past [5-7].

Health professionals play a major role in providing health services. Internet usage among health professionals helps to update themselves about the recent advances in the health care system so that they can deliver appropriate health services to the people. The Internet is a popular source of health information for health care providers and consumers. It has been recognized by many as an important mechanism for transforming medical care. The internet affords healthcare practitioners unprecedented access to huge volume, high quality, current and relevant health care information. Among currently available technologies only the Internet has the potential to deliver universal access to up-to-date health care information [8]. Accurate and up-to-date information is vital to maintain quality of health care. During the past decade, many health information resources have been developed and available online for use by health care professionals.

Rehman et al., conducted a study on the Internet use by health professionals at the health sciences centre (HSC) of Kuwait University [9]. The study indicated that $80.3 \%$ of the respondents used Internet daily. $88.2 \%$ of the respondents felt that the Internet provided better access to health sciences information, $77.2 \%$ indicated that through the Internet they had better professional contacts and $57.5 \%$ stated that with the use of Internet they were able to use different channels of communication for their patient care and research. A study conducted by Ajuwon to know the physicians' use of the Internet for health information for patient care at the University College Hospital (UCH) Ibadan, Nigeria. The findings revealed that $98 \%$ of the respondents had used the Internet. Ninety percent reported that they had obtained information from the Internet for patient care, $76.2 \%$ had searched a database.

A study conducted by Reynolds et al., among 457 dentists in Wales to identify how patient information on the Internet has influenced the delivery of oral care and the use practitioners make of the Internet [10]. Thirty-nine percent of respondents agreed that information gained from the Internet had led to patients demanding inappropriate care.

As far to the best of our knowledge, many studies highlighted the use of internet among patients in seeking health information but there are only few studies among health care professionals who use internet for seeking health related information. Hence this study was undertaken with an aim to investigate the usage of internet in seeking health related information among health care practitioners.

\section{METHODOLOGY}

A cross sectional questionnaire based study was conducted for a period of one month to assess the usage of internet in seeking health related information among medical and dental health practitioners in Hyderabad city. Ethical clearance was obtained from the institutional review board of Sri Sai College of Dental Surgery (Ref No.222/SSCDS/IRB-E/2016). Pilot study was done to determine the feasibility of the study and assess the sample size. A total of 400 medical and dental health practitioners from all the five zones who were practicing in Hyderabad city were included in the study.

A pretested structured 15 item questionnaire was used to collect the data. The questionnaire was developed based on the previous literature. The first part of the questionnaire included demographics and second part included questions regarding health professional's motives to use internet for seeking health information and third part included questions regarding barriers to use internet. Pilot study was conducted among 30 health practitioners to check the feasibility. The responses of the questionnaire was rated on a 3 point likert scale ranged from agree to neutral. Validity and reliability of the questionnaire was checked (crohnbach's alpha 0.07). Data was collected from all the five zones of Hyderabad city by area sampling method. Collected data was subjected to statistical analysis.

\section{Statistical Analysis}

Descriptive analysis was done to know the usage of internet among health practitioners. Chi square test was done to compare the usage of internet among medical and dental practitioners. The level of significance was set at $5 \%$. The data were analyzed with the Statistical Package for Social Sciences (SPSS 21).

\section{RESULTS}

A total of 200 medical and 200 dental practitioners completed the study. Among those $57 \%$ were males and $43 \%$ of them were females and mean age of the participants was 35 years.

Among health practitioners motives to use internet, $61(15.3 \%)$ of the participants reported that internet is a platform to share and receive information. $190(47.5 \%)$ of them agreed that they use internet to get closer contacts with community. 149(37.3\%) of the participants agreed that they use internet for clarification of doubts in the diagnosis of the disease. $77(19.3 \%)$ of them reported that they use internet for getting updated about the recent treatment procedures and $85(21.3 \%)$ agreed that for getting updates about the 
new materials they use internet. 166(41.5\%) of the participants reported that they use internet to study the disease patterns across various geographic locations. $133(33.3 \%)$ of them agreed they use internet to attend online courses and $83(20.8 \%)$ of them reported that they use internet to share information about employment and research opportunities (Table-1).

When health practitioners barriers to use internet was assessed $166(41.5 \%)$ of the participants agreed that lack of skills, $200(50 \%)$ agreed that inaccessibility of required information, $159(39.8 \%)$ reported lack of time and $114(28.5 \%)$ of them agreed that difficulty to regulate the quality of care were the barriers to use internet (Table-2).

Our findings showed a significant difference in the usage of internet among medical and dental practitioners in which most of the medical practitioners used internet for getting updates in treatment procedures, to make closer contacts with the community, to share employment and research opportunities whereas dental practitioners used internet for getting updated about the new materials, to study various disease patterns, to share and receive information and to attend online courses (Table-3).

Table-1: Distribution of medical and dental practitioner's responses in usage of internet

\begin{tabular}{|l|l|l|l|}
\hline Question & Agree & Disagree & Neutral \\
\hline To share and receive information & $61(15.3)$ & $19(4.8)$ & $320(80.0)$ \\
\hline To get in closer contacts with community & $190(47.5)$ & $27(6.8)$ & $183(45.8)$ \\
\hline For diagnosis of disease & $149(37.3)$ & $28(7.0)$ & $223(55.8)$ \\
\hline Recent advances treatment procedure & $77(19.3)$ & $9(2.3)$ & $314(78.5)$ \\
\hline Updates about new materials & $85(21.3)$ & $12(3.0)$ & $303(75.8)$ \\
\hline To study about various patterns of diseases across different locations & $166(41.5)$ & $29(7.2)$ & $205(51.2)$ \\
\hline To track patient experiences and quality of care & $156(39.0)$ & $36(9.0)$ & $208(52.0)$ \\
\hline For attending online courses & $133(33.3)$ & $46(11.5)$ & $221(55.3)$ \\
\hline As a teaching tool & $110(27.5)$ & $41(10.3)$ & $249(62.3)$ \\
\hline Share information about employment and research opportunities & $83(20.8)$ & $37(9.3)$ & $280(70.0)$ \\
\hline
\end{tabular}

Table-2: Medical and Dental practitioner's barriers to use internet

\begin{tabular}{|l|l|l|l|}
\hline BARRIER & Agree & Disagree & Neutral \\
\hline Lack of skills & $166(41.5)$ & $17(4.3)$ & $217(54.3)$ \\
\hline I did not find any need to use & $261(65.3)$ & $23(5.8)$ & $116(29.0)$ \\
\hline Inaccessibility of required information & $200(50.0)$ & $19(4.8)$ & $181(45.3)$ \\
\hline Lack of time & $159(39.8)$ & $23(5.8)$ & $218(54.5)$ \\
\hline Difficulty to regulate the quality of care & $114(28.5)$ & $25(6.3)$ & $261(65.3)$ \\
\hline
\end{tabular}

Table-3: Comparison of medical and dental practitioners motives to use internet

\begin{tabular}{|l|l|l|l|l|}
\hline Questions & Agree & Disagree & Neutral & P value \\
\hline To share and receive information & $61(15.3)$ & $19(4.8)$ & $320(80.0)$ & 0.00 \\
\hline To get in closer contacts with community & $190(47.5)$ & $27(6.8)$ & $183(45.8)$ & 0.05 \\
\hline For diagnosis of disease & $149(37.3)$ & $28(7.0)$ & $223(55.8)$ & 0.00 \\
\hline Recent advances treatment procedure & $77(19.3)$ & $9(2.3)$ & $314(78.5)$ & 0.00 \\
\hline Updates about new materials & $85(21.3)$ & $12(3.0)$ & $303(75.8)$ & 0.00 \\
\hline To study about various patterns of diseases across different locations & $166(41.5)$ & $29(7.2)$ & $205(51.2)$ & 0.08 \\
\hline To track patient experiences and quality of care & $156(39.0)$ & $36(9.0)$ & $208(52.0)$ & 0.07 \\
\hline For attending online courses & $133(33.3)$ & $46(11.5)$ & $221(55.3)$ & 0.05 \\
\hline As a teaching tool & $110(27.5)$ & $41(10.3)$ & $249(62.3)$ & 0.08 \\
\hline Share information about employment and research opportunities & $83(20.8)$ & $37(9.3)$ & $280(70.0)$ & 0.05 \\
\hline
\end{tabular}

Chi square test $p<0.05$ is statistically significant.

\section{DISCUSSION}

The Internet as a medium of communication is useful in medicine; and has become an important means of how health professionals deliver health care [11]. Information technology is recognized as a useful tool for improving patient safety and quality of care. The Internet has the potential to improve information dissemination and change the way health care is delivered. Internet seems to provide a new opportunity to overcome problems of access and provide clinically appropriate information to practitioners. However, while use of Internet for clinical information has grown substantially in recent years. Access to the Internet has been improving rapidly for all professional groups [12].

In present study majority of them were males with mean age group of 37 years and most of them were general practitioners who has 6-10 years of experience with no significant difference of using internet. All the respondents in this study (98\%) had used the 
Internet. This finding is comparable to a study done by Grace A Ajuwon ${ }^{3}$ were $98 \%$ of resident physicians used internet for health information seeking in University of Pennsylvania Medical School. This finding is evidence of how ubiquitous the Internet has become even in tertiary institutions in a developing country; although Internet access is much less available at primary and district levels.

In this study most of the respondents indicated that they use Google as the major source of internet platform which is in contrast with a study conducted by Marjolijin Antheunis et al., [2] in which the health professionals frequently used facebook for seeking health information and majority of health practitioners in this study reported that they use internet daily for seeking health information. This might be because as the Internet is a fast means of communication and contains more varied information than one could ever imagine most of the prefer to use daily.

The Internet is considered to be a significant source of health in-formation for the general public. It allows increased access to medical and dental information and can be used for patient self-education, enabling patients to confirm the information given by their practitioner and to gather additional information Physicians and dentists have diverse information needs when making informed diagnoses and treatment decisions. While medical knowledge continues to grow at a steady pace, clinicians are spending less time with patients, leading to lower patient satisfaction. As a result, identifying information clinicians need at the point of care and accurately and efficiently providing it have become issues of critical importance [12].

When dental practitioner's motives to use internet for seeking health related information was assessed majority of them agreed that it is a platform to share and receive information. This finding is similar to a study conducted by Hana Alsobayel 2 in which $75 \%$ of the health professionals agreed that internet is used for exchange of knowledge. Majority of them agreed that they use internet for getting updated about the recent advances in both diagnosis and preventive, curative treatment procedures and for getting information about the new materials, to study various disease patterns across different locations. This might be due to high cost of books and subscription-based journals, only few individuals can afford to subscribe to journals while the majority relies on the medical library for needed information. This development has resulted in health professionals relying more on the Internet for health information. Also, the Open Access initiative has made it possible for articles published in some journals to be accessed freely on the Internet [4].

Health practitioners need information management skills to cope with the growing volume of medical knowledge. The ability to quickly access, track, coordinate, critique, and store information is essential to informed daily decision making related to patient care, education, and research [12]. In the present study when medical practitioner's motives to use used internet was assessed majority of them agreed that they use internet to get updated about the recent advances in treatment procedures. This might be because as most the medical practitioners deals with major treatment procedures so they should be updated to provide good quality of care. Majority of them agreed that they use internet frequently to share employment and research opportunities which is similar to a study conducted by Hana Alsobayel [3] and majority of the participants also agreed that they use internet to attend online courses.

When medical and dental practitioners motives to use internet was compared a significant difference was found in the usage of internet in which medical practitioners used internet frequently for getting updated about the recent advances in treatment procedures and for clarification of doubts whereas dental practitioners used frequently to get updated about the new materials and techniques and also for knowledge exchange

This study also assessed health practitioner's barriers to use internet and the major barriers reported was difficulty in regulating the quality of care and inaccessibility of the required information.

Since the impact of the internet and other technological developments on health care is expected to increase health professionals will have to keep pace with the potential effects of internet usage on clinical practice.

\section{CONCLUSION}

This study concludes that all the health care professionals participating in this study used internet for seeking health information but there was a significant difference among the medical and dental health professionals in usage of internet in which most of the medical practitioners used internet for getting updated about the treatment procedure whereas dental practitioners used for getting updated about the advances in new materials and techniques.

\section{RECOMMENDATIONS}

In the past, there was no problem in identifying the information sources in libraries as the major information carriers were mainly in printed format. But the advent of Internet as a viable information and communication technology (ICT) tools has facilitated access to health information resources which has cause a paradigm shift in the information seeking behaviour of users [13-15]. Therefore, understanding the information seeking behaviour of health professionals will help librarian to provide better and improved health information resources. The following recommendations can be made [16-18]: 
- There should be training for health professionals on the use of Internet health information resources and databases in the library.

- There should be improvement of electronic technologies by the management as regards to connection speed, efficient navigability, and overall usability).

- There should be an effective subscription of health-related databases, and awareness of open access journals, clinical software applications etc, to increase wider access of health information resources.

\section{REFERENCES}

1. Dennisson, J. P., Hope, T., Perrella, R., Schaub, R. L., \& Slemmer, J. B. (2010). U.S. Patent No. 7,650,111. Washington, DC: U.S. Patent and Trademark Office.

2. Antheunis, M. L., Tates, K., \& Nieboer, T. E. (2013). Patients' and health professionals' use of social media in health care: motives, barriers and expectations. Patient education and counseling, 92(3), 426-431.

3. Alsobayel, H. (2016). Use of social media for professional development by health care professionals: a cross-sectional webbased survey. JMIR medical education, 2(2), e15.

4. Ajuwon, G. A. (2006). Use of the Internet for health information by physicians for patient care in a teaching hospital in Ibadan, Nigeria. Biomedical Digital Libraries, 3(1), 12.

5. Apostolakis, I., Koulierakis, G., Berler, A., Chryssanthou, A., \& Varlamis, I. (2012). Use of social media by healthcare professionals in Greece: an exploratory study. International journal of electronic healthcare, 7(2), 105-124.

6. Usher, W. T. (2012). Australian health professionals' social media (Web 2.0) adoption trends: early 21st century health care delivery and practice promotion. Australian Journal of Primary Health, 18(1), 31-41.

7. Barry, A. R., \& Pearson, G. J. (2015). Professional use of social media by pharmacists. The Canadian journal of hospital pharmacy, 68(1), 22-27.

8. Derseh, M. A. (2013). Implementation of Anti-Retroviral Therapy (ART) pharmacy management information system in health facilities in Ethiopia (Doctoral dissertation).

9. Rehman, J., Li, J., Orschell, C. M., \& March, K. L. (2003). Peripheral blood "endothelial progenitor cells" are derived from monocyte/macrophages and secrete angiogenic growth factors. Circulation, 107(8), 1164-1169.

10. Reynolds, W. M., \& Mazza, J. J. (1998). Reliability and validity of the Reynolds Adolescent Depression Scale with young adolescents. Journal of School Psychology, 36(3), 295-312.

11. Neville, P., \& Waylen, A. (2015). Social media and dentistry: some reflections on e-professionalism. British dental journal, 218(8), $475-478$.

12. Mayur, N., \& Hemalath, T. (2018). Use of online sources of information for dental practice among private dental practitioners in Bangalore - A cross sectional survey. International Journal of Dental Resarch, 5(1): 75-82.

13. D'Souza, K., Henningham, L., Zou, R., Huang, J., O'Sullivan, E., Last, J., \& Ho, K. (2017). Attitudes of health professional educators toward the use of social media as a teaching tool: global cross-sectional study. JMIR medical education, 3(2), e13.

14. Archambault, P. M., Légaré, F., Lavoie, A., Gagnon, M. P., Lapointe, J., St-Jacques, S., ... \& Pham-Dinh, M. (2010). Healthcare professionals' intentions to use wiki-based reminders to promote best practices in trauma care: a survey protocol. Implementation Science, 5(1), 45-52.

15. Bhaskaran, N., Kumar, M., \& Janodia, M. D. (2017). Use of Social Media for Seeking Health Related Information-An Exploratory Study. Journal of Young Pharmacists, 9(2): 267-271.

16. Dhatt, K. S., \& Kaliaperumal, C. (2014). Incorporation of web-based applications and online resources in undergraduate medical education in the Irish Republic. Can new changes be incorporated in the current medical curriculum?. Journal of natural science, biology, and medicine, 5(2), 445-449.

17. Christensen, H., Murray, K., Calear, A. L., Bennett, K., Bennett, A., \& Griffiths, K. M. (2010). Beacon: a web portal to high-quality mental health websites for use by health professionals and the public. Medical Journal of Australia, 192, S40-S44.

18. López-Jornet, P., \& Camacho-Alonso, F. (2010). The quality of patient-orientated Internet information on oral lichen planus: a pilot study. Journal of evaluation in clinical practice, 16(5), 883-886. 